\section{[gw22-e0488] PU-ERH TEA CAN INHIBIT ATHEROSCLEROSIS BY PROMOTING MACROPHAGE APOPTOSIS IN PLAQUE THROUGH NF-KB PATHWAY IN APOE NULL MICE}

He Ming, Liang Xiao, Wen Wen, Zhao Sijia, Chen Tao, Liu Yan Department Of Cardiology, First Affiliated Hospital Of Medical School, Xi'an Jiaotong University, Shaanxi, China

10.1136/heartjnl-2011-300867.46

Purpose Pu-erh tea has recently become very popular in China and most southeast Asian countries. Fermented Pu-erh tea may help regulate blood lipids, prevent and treat atherosclerosis (AS). Below, we discuss the effects of Pu-erh tea on AS.

Methods Blood lipid levels, plaque size and composition, proinflammatory cytokines expression and NF- $\mathrm{KB}$ binding activity were determined in ApoE null mice that consumed Pu-erh tea for either eight or 16 weeks. RAW264.7 cells were treated with $\mathrm{Pu}$-erh tea water extract in vitro.

Results In vivo, there were no significant differences in blood lipid levels between the tea consumption groups and controls. Among the ApoE null mice who consumed pu-erh tea for 16 weeks, the mean plaque area decreased by $18.66 \%$, the mean ratios of oil red $\mathrm{O}$ positive regions decreased by $18.05 \%$, the mean proportion of macrophages in the plaque decreased by $41.29 \%$ and the apoptotic macrophages in plaque increased 2.69-fold. NF- $\mathrm{\kappa B}$ binding activity in peritoneal macrophages decreased by $75.55 \%$ and the relative mRNA expression levels of IL-6, IL-12 and TNF $\alpha$ in the vascular tissue decreased by $17.83 \%, 36.39 \%$ and $43.73 \%$, respectively. In vitro, The treatment of RAW264.7 cells with pu-erh tea water extract found the expression of the P65 could be reduced, whereas IкB $\beta$ expression in the cells increased. Using EMSA to detect the binding activity of NF- $\mathrm{kB}$, increasing treatment concentrations decreased the mean grey value significantly and depending on the length of treatment time. With extended intervention time and increased tea water extract concentrations, the mRNA expression levels of IL-6 decreased. When RAW264.7 were treated with a certain concentration of pu-erh tea water extract, within the first $24 \mathrm{~h}$ and with the extension of the intervention time, the proportion of apoptotic cells continued to increase, and within increased concentrations of pu-erh tea extract for $24 \mathrm{~h}$, the proportion of apoptotic cells continued to rise. Pretreated with PMA, the proportion of positive apoptotic cells decreased with most points, with the lowest point at $62.16 \%$ decreased; pretreated with Bay11-7085, the proportion of positive apoptotic cells increased, reaching a high point of $23.39 \%$ increased. After adding different concentrations of pu-erh tea water extract, no changes in the RAW264.7 cells' migration ability was observed. Pu-erh Tea water extract had no effect on macrophage foam cell formation, cholesterol concentration and efflux rate, mRNA expression of ABCA1, ABCG1 and CD36 of RAW264.7 cells.

Conclusion Pu-erh tea is able to inhibit a chronic inflammatory state of the body and local blood vessels (take the decrease of the expression of TNF $\alpha$ and IL- 6 as a sign) and reduce the activity of NF- $\mathrm{\kappa B}$ expression, thus promoting macrophage apoptosis in the plaque, reducing the number of macrophages in plaque, and the plaque area and lipid deposition, 
and ultimately delaying the progress of AS. Pu-erh tea had no effect on the circulating cholesterol level, the macrophage migration into the plaque, macrophages' engulfing of cholesterol and their reverse transport of cholesterol. Our research shows that in ApoE null mice, pu-erh tea does impede the progress of AS, we are convinced that drinking pu-erh tea can effectively stop the progress of AS, and this lifestyle habit in Asian countries should be promoted worldwide, especially in areas with a high prevalence of AS. 